Check for updates

Cite this: RSC Adv., 2019, 9, 10155

Received 23rd January 2019

Accepted 26th March 2019

DOI: 10.1039/c9ra00595a

rsc.li/rsc-advances

\title{
Controlled synthesis of uniform multilayer hexagonal boron nitride films on $\mathrm{Fe}_{2} \mathrm{~B}$ alloy
}

\author{
Zhiyuan Shi, ${ }^{\text {abc }}$ Guangyuan Lu, ${ }^{\text {ac }}$ Peng Yang, ${ }^{d}$ Tianru Wu, (D)*ac Weijun Yin, ${ }^{\text {a }}$ \\ Chao Zhang, ${ }^{\text {abc }}$ Ren Jiang ${ }^{\text {ae }}$ and Xiaoming Xie ${ }^{a b c f}$
}

Two-dimensional (2D) hexagonal boron nitride ( $\mathrm{h}-\mathrm{BN})$ is highly appreciated for its excellent insulating performance and absence of dangling bonds, which could be employed to maintain the intrinsic properties of $2 \mathrm{D}$ materials. However, controllable synthesis of large scale multilayer $\mathrm{h}-\mathrm{BN}$ is still very challenging. Here, we demonstrate chemical vapor deposition (CVD) growth of multilayer h-BN by using iron boride $\left(\mathrm{Fe}_{2} \mathrm{~B}\right)$ alloy and nitrogen $\left(\mathrm{N}_{2}\right)$ as precursors. Different from the self-limited growth mechanism of monolayer $h-B N$ on catalytic metal surfaces, with sufficient $B$ source in the bulk, $\mathrm{Fe}_{2} B$ alloy promotes the controllable isothermal segregation of multilayer $h-B N$ by reacting with active $N$ atoms on the surface of the substrate. Microscopic and spectroscopic characterizations prove the high uniformity and crystalline quality of $\mathrm{h}-\mathrm{BN}$ with a highly orientated layered lattice structure. The achievement of large scale multilayer $\mathrm{h}-\mathrm{BN}$ in this work would facilitate its applications in 2D electronics and optoelectronics in the future.

\section{Introduction}

Two-dimensional (2D) materials have attracted enormous attention for future electronic and optoelectronic device integrations. ${ }^{1-3}$ Hexagonal boron nitride (h-BN), a 2D insulator with a wide band gap $(\sim 6 \mathrm{eV})$, is in general highly desired for practical device applications, such as gate dielectric layers, tunnelling barriers or encapsulations. ${ }^{4-7}$ It is widely regarded as an ideal dielectric substrate for 2D materials because of its atomic flatness and absence of dangling bonds, which could maintain the intrinsic properties of the overlying 2D materials., ${ }^{3,5,6,8}$ Moreover, single phonon emission from isolated point defect sites in h-BN has been investigated recently, which demonstrates potential in quantum photonics and quantum computation. ${ }^{9-11}$

So far, many efforts have been devoted to synthesize highquality h-BN films. In recent years, layered h-BN flakes,

${ }^{a}$ State Key Laboratory of Functional Materials for Informatics, Shanghai Institute of Microsystem and Information Technology, Chinese Academy of Sciences, Shanghai 200050, China.E-mail: trwu@mail.sim.ac.cn

${ }^{b}$ School of Electronic, Electrical and Communication Engineering, University of Chinese Academy of Sciences, 19 A Yuquan Road, Beijing 100049, China

${ }^{c}$ CAS Center for Excellence in Superconducting Electronics (CENSE), Shanghai 200050, China

${ }^{d}$ State Key Laboratory of ASIC and System, School of Information Science and Technology, Fudan University, 220 Handan Road, Shanghai 200433, China

eState Key Laboratory of Precision Spectroscopy, School of Physics and Material Science, East China Normal University, 3663 N. Zhongshan Road, Shanghai 200062, China

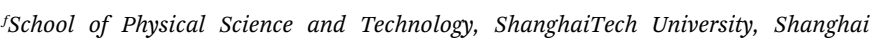
200031, China integrated with other layered materials for $2 \mathrm{D}$ heterostructure devices, were mechanically exfoliated from high quality h-BN bulk crystal, which was obtained under extremely high temperature and pressure for several days. ${ }^{12,13}$ However, the limited size and inhomogeneous thickness hinder further development of 2D electronics and photonics. As the most promising technique, chemical vapor deposition (CVD) was employed to achieve large-area monolayer and few layer h-BN on metal or dielectric substrates by using ammonia borane $\left(\mathrm{NH}_{3} \cdot \mathrm{BH}_{3}\right)$ or borazine $\left(\mathrm{B}_{3} \mathrm{~N}_{3} \mathrm{H}_{6}\right)$ as the precursor. ${ }^{14-23}$ However, the inhomogeneous thickness, poor crystallinity, toxicity of precursors and complicated procedures limits the application in practical 2D devices. High quality, thickness uniformity, low cost and facile synthesis remain extremely challenging for compound 2D layered materials such as h-BN.

Here, we report a new method to synthesize multilayer h-BN on $\mathrm{Fe}_{2} \mathrm{~B}$ alloy with $\mathrm{N}_{2}$ as a reactant. It is much easier to control the flow rate by using gaseous $\mathrm{N}_{2}$ as a precursor than solid precursors $\left(\mathrm{NH}_{3} \cdot \mathrm{BH}_{3}\right.$ or $\left.\mathrm{B}_{3} \mathrm{~N}_{3} \mathrm{H}_{6}\right)$. Comparing with previous approaches, the new synthesis method yields large scale and high quality multilayer h-BN at ambient pressure. The surface morphology, crystalline quality and defect distributions were investigated by various characterizations. $\mathrm{Fe}_{2} \mathrm{~B}$, an alloy not only supplies boron source directly, but also reacts with $\mathrm{N}_{2}$ to form multilayer h-BN. The entire growth process breaks the traditional self-limited growth mechanism on catalytic metal substrates and successfully obtains multilayer h-BN on $\mathrm{Fe}_{2} \mathrm{~B}$ alloy. Controllable synthesis of h-BN film establishes a significant approach for further rational catalyst design for other compound 2D materials. 


\section{Materials and methods}

Multilayer h-BN was synthesized on $\mathrm{Fe}_{2} \mathrm{~B}$ alloy using ambient pressure CVD. Firstly, a $\mathrm{Fe}_{2} \mathrm{~B}$ substrate was heated to $1300{ }^{\circ} \mathrm{C}$ and maintained for 60 minutes under $\mathrm{Ar}$ and $\mathrm{H}_{2}$ atmosphere at flow rates of 300 and $50 \mathrm{sccm}$, respectively. Then, $\mathrm{N}_{2}$ and $\mathrm{H}_{2}$ atmosphere at flow rates of $300 \mathrm{sccm}$ and $50 \mathrm{sccm}$ were introduced at $1300{ }^{\circ} \mathrm{C}$ for 60 minutes to facilitate h-BN thin film growth. Finally, the furnace was cooled down to room temperature naturally under $\mathrm{Ar}$ and $\mathrm{H}_{2}$ flows.

The as-grown multilayer h-BN films were transferred to arbitrary substrates through chemical transfer process. The morphology of multilayer h-BN on $\mathrm{Fe}_{2} \mathrm{~B}$ alloy was characterized by scanning electron microscope (SEM, Zeiss Supra 55, operated at $3 \mathrm{kV}$ ). Atomic force microscope (AFM, Bruker Icon, Tapping mode) was employed to determine the thickness of multilayer $h$ BN. Raman spectra were obtained in the spectral range of 1100$1700\left(\mathrm{~cm}^{-1}\right)$ by using a laser excitation of $532 \mathrm{~nm}$. The stoichiometry of B and $\mathrm{N}$ of h-BN was evaluated by X-ray photoemission spectroscopy (XPS, species, $\mathrm{Al} \mathrm{K \alpha}$ ). The phase structure of h-BN thin film was identified by X-ray diffraction (XRD, Bruker discover D8). Transmission electron microscope (TEM, JEM-2100F, operated at $200 \mathrm{kV}$ ) images and selected area electron diffraction (SAED) confirm the high crystalline quality of as-prepared h-BN. Photoluminescence (PL) mapping and PL spectra were collected by using a laser excitation of $532 \mathrm{~nm}$ (WITec Alpha 300R, $5 \mathrm{~mW}$ ).

\section{Results and discussion}

The schematic of ambient-pressure CVD setup for multilayer hBN growth was depicted in Fig. 1a (details are given in Materials and methods). To demonstrate the growth dynamics, $\mathrm{Fe}_{2} \mathrm{~B}$ alloys were annealed at $1100{ }^{\circ} \mathrm{C}, 1200{ }^{\circ} \mathrm{C}$ and $1300{ }^{\circ} \mathrm{C}$ for 60 minutes, respectively. SEM images exhibit h-BN nucleus, isolated h-BN flakes and continuous h-BN film synthesized at
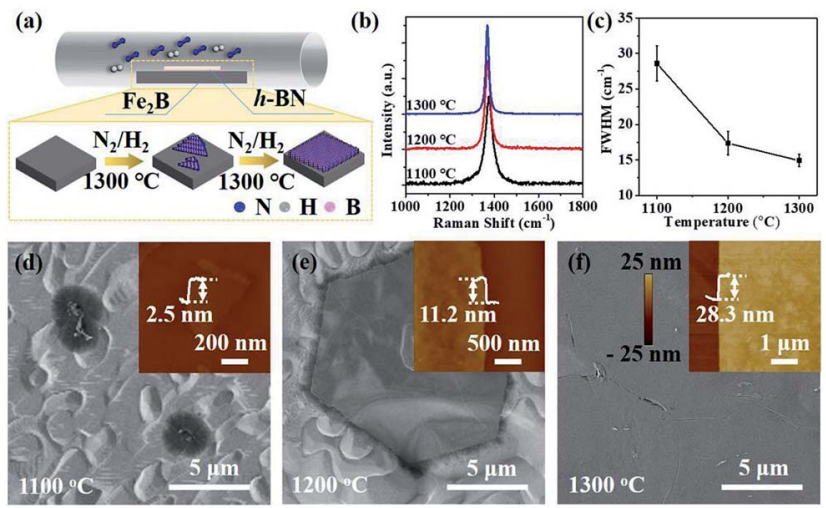

Fig. 1 (a) Schematic diagram for synthesis of multilayer $\mathrm{h}-\mathrm{BN}$ on $\mathrm{Fe}_{2} \mathrm{~B}$ alloy. (b) Raman spectra of $\mathrm{h}-\mathrm{BN}$ grown on $\mathrm{Fe}_{2} \mathrm{~B}$ alloy at $1100{ }^{\circ} \mathrm{C}$, $1200^{\circ} \mathrm{C}$ and $1300^{\circ} \mathrm{C}$, respectively. (c) The relationship between FWHM of $E_{2 g}$ phonon mode and growth temperatures. (d-f) SEM images of $h$ $\mathrm{BN}$ grown on Fe-B alloy at $1100{ }^{\circ} \mathrm{C}$ (d), $1200{ }^{\circ} \mathrm{C}$ (e) and $1300^{\circ} \mathrm{C}$ (f), respectively. Corresponding $\mathrm{AFM}$ images with height profiles were displayed in the inset. different growth temperatures (Fig. 1d-f). Corresponding AFM images and height profiles in the inset verify the thickness of asgrown h-BN transferred on $\mathrm{SiO}_{2}(300 \mathrm{~nm}) / \mathrm{Si}$ substrate. By introducing $\mathrm{N}_{2}$ gas as a reactant, uniform multilayer h-BN films over large areas formed on $\mathrm{Fe}_{2} \mathrm{~B}$ substrate. Fig. $1 \mathrm{~b}$ displays the Raman spectra of multilayer h-BN films prepared at various growth temperatures. It could be observed that the position of characteristic $\mathrm{E}_{2 \mathrm{~g}}$ band presents a slightly shift (from 1372 to $1366 \mathrm{~cm}^{-1}$ ) with the increasing of growth temperature, indicating the increase of thickness. ${ }^{24}$ According to earlier reports, the full-width at half maximum (FWHM) of the Raman $\mathrm{E}_{2 \mathrm{~g}}$ band is associated with the crystallinity of h-BN. ${ }^{24-26}$ The FWHM of $\mathrm{E}_{2 \mathrm{~g}}$ phonon mode decreased with growth temperature increasing (Fig. 1c). The h-BN synthesized at $1300{ }^{\circ} \mathrm{C}$ shows the minimum FWHM value of $\sim 15 \mathrm{~cm}^{-1}$, which is much smaller than that synthesized by existing catalytic epitaxial approaches. $^{25}$

Multilayer h-BN film obtained at $1300{ }^{\circ} \mathrm{C}$ was further characterized to analyse its morphology and crystalline quality. Continuous and uniform h-BN film grown on $\mathrm{Fe}_{2} \mathrm{~B}$ alloy was displayed in Fig. 2a. Layered structure was verified primarily by measuring the edge of h-BN film. To further evaluate the thickness uniformity of h-BN films on $\mathrm{Fe}_{2} \mathrm{~B}$ substrate, the asgrown multilayer h-BN was transferred on $\mathrm{a} \mathrm{SiO}_{2}(300 \mathrm{~nm}) / \mathrm{Si}$ substrate. The uniform optical contrast confirms the smooth surface and high uniformity of thickness (Fig. 2b). The thickness of the film is around $30 \mathrm{~nm}$ according to the height profile on the edge of h-BN (Fig. 2c). Also, Raman mapping of $\mathrm{E}_{2 \mathrm{~g}}$ phonon mode depicts the homogeneity thickness of asprepared h-BN multilayers (Fig. 2d). Moreover, we investigated the composition and crystal structure of h-BN film. The survey XPS spectrum of h-BN film, displayed in Fig. 2e, indicates that the stoichiometry of $\mathrm{B}$ and $\mathrm{N}$ atoms is $1: 1.06$. Further, The XRD spectrum displays sharp (0002) diffraction peak at $26.8^{\circ}$ and (0004) diffraction peak at $55.2^{\circ}$, which signifies the layers of $h$ $\mathrm{BN}$ are well aligned with the $c$ axis (Fig. 2f). ${ }^{18}$ The Inorganic Crystal Structure Database (ICSD) (h-BN, 340421) was used for

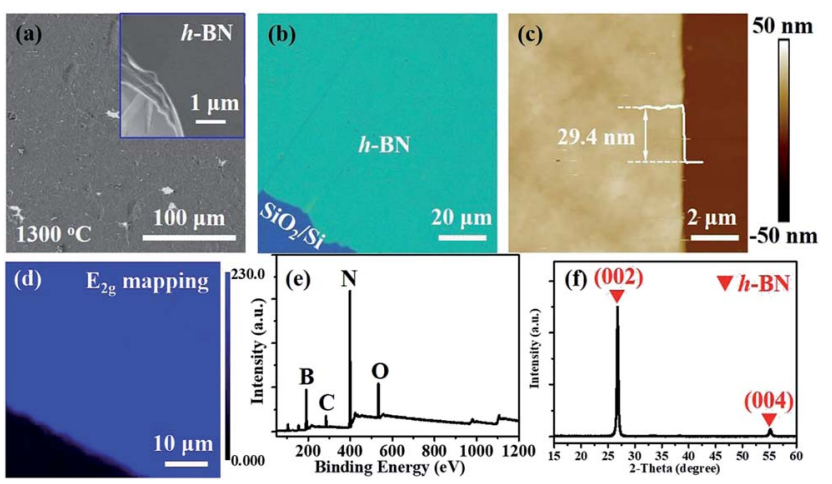

Fig. 2 (a) SEM image of multilayer h-BN film grown on Fe-B alloy at $1300^{\circ} \mathrm{C}$. Inset shows the edge of $\mathrm{h}-\mathrm{BN}$ film. (b) OM image of multilayer h-BN transferred onto $\mathrm{SiO}_{2}(300 \mathrm{~nm}) / \mathrm{Si}$ substrate. (c) AFM image and corresponding height profile of $\mathrm{h}-\mathrm{BN}$ film. (d) Raman mapping of $\mathrm{E}_{2 \mathrm{~g}}$ phonon mode of $h-B N$ film. (e and f). Survey XPS (e) and XRD (f) spectra of h-BN film. 
phase identification. The lattice constant was calculated to be $0.33 \mathrm{~nm}$, which is in good agreement with reported values. ${ }^{18}$

As previous reports, $\mathrm{NH}_{3} \cdot \mathrm{BH}_{3}$ or $\mathrm{B}_{3} \mathrm{~N}_{3} \mathrm{H}_{6}$ was always used to synthesize monolayer or few-layer h-BN on metal substrates. ${ }^{\mathbf{1 4 - 1 7 , 1 9 , 2 1 , 2 3}}$ For monolayer h-BN grown on $\mathrm{Cu}-\mathrm{Ni}$ alloy, $\mathrm{B}_{3} \mathrm{~N}_{3} \mathrm{H}_{6}$ precursor undergoes decompose, polymerization and dehydrogenation on the surface of the substrate (Fig. 3d). ${ }^{19}$ SEM image displays the morphology of h-BN domains and TEM image signifies the monolayer nature of as-grown h-BN (Fig. $3 f$ and $\mathrm{g}$ ). The surfaces do not assist the synthesis of multilayer h$\mathrm{BN}$ due to the self-limited growth effect when monolayer h-BN covered the $\mathrm{Cu}-\mathrm{Ni}$ alloy. ${ }^{19}$ For multilayer h-BN grown on $\mathrm{Fe}_{2} \mathrm{~B}$ alloy, $\mathrm{N}_{2}$ would firstly decompose into active nitrogen atoms with the catalysis of $\mathrm{Fe}_{2} \mathrm{~B}$ substrate. ${ }^{27}$ Then, active nitrogen atoms would react with $\mathrm{Fe}_{2} \mathrm{~B}$ to form initial $\mathrm{B}-\mathrm{N}$ molecular. ${ }^{18,27}$ High concentration $\mathrm{B}-\mathrm{N}$ molecules would segregate and induce the nucleation of h-BN isothermally. Finally, h-BN nucleus would grow and coalesce into multilayer h-BN films on the surface of $\mathrm{Fe}_{2} \mathrm{~B}$ alloy. The controllable thickness would be realized by optimizing growth temperature or reaction time (Fig. 3a). SEM image of as-grown multilayer h-BN displays the uniform and smooth surface morphology and TEM image indicates multi-layered h-BN nanostructure (Fig. $3 \mathrm{~b}$ and c). Further, $\mathrm{E}_{2 \mathrm{~g}}$ phonon mode of multilayer h-BN in the Raman spectrum shows much stronger intensity than the $\mathrm{E}_{2 \mathrm{~g}}$ band of monolayer h-BN (Fig. 3e). Comparing with the two synthesis approaches mentioned above, the success of thickness controllable growth is mainly due to the change of h-BN growth mechanism from surface-mediated growth to isothermal segregation on $\mathrm{Fe}_{2} \mathrm{~B}$ substrate by using nitrogen as feedstock.

The crystalline quality and atomic structure of the multilayer h-BN is further characterized by TEM. The low-magnified TEM view in Fig. 4a displays continuous and uniform h-BN film suspended on $\mathrm{Cu}$ mesh grid. High resolution TEM image exhibits the lattice fringe of h-BN multilayers (Fig. 4b). Corresponding selected area electron diffraction (SAED) presents only one set of hexagonally arranged spots, rather than ringshaped patterns, assuring the highly oriented stacking order. ${ }^{20}$ Moreover, Fig. 4c reveals a honeycomb structure with an interatomic distance of about $0.25 \mathrm{~nm}$, in good agreement with

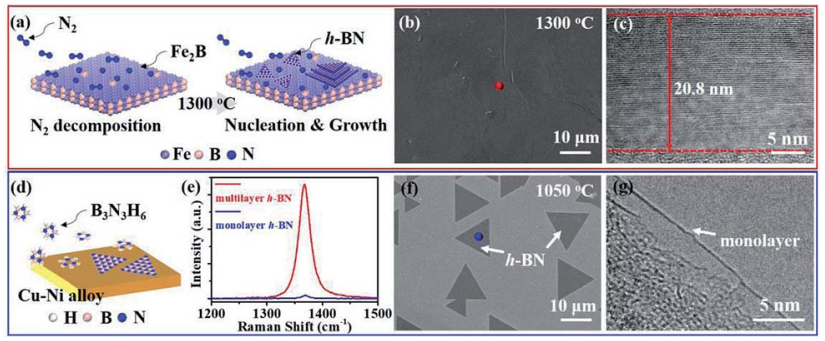

Fig. 3 (a) Schematic of the growth process of multilayer $h-B N$ grown on $\mathrm{Fe}_{2} \mathrm{~B}$ alloy with $\mathrm{N}_{2}$ as a precursor. (b and c) Typical SEM (b) and TEM (c) images of as-grown multilayer $\mathrm{h}-\mathrm{BN}$. (d) Schematic illustration of the growth process of monolayer $\mathrm{h}-\mathrm{BN}$ on $\mathrm{Cu}-\mathrm{Ni}$ alloy with $\mathrm{B}_{3} \mathrm{~N}_{3} \mathrm{H}_{6}$ as a precursor. (e) Raman spectra of multilayer (red line) and monolayer (blue line) $\mathrm{h}-\mathrm{BN}$. ( $\mathrm{f}$ and $\mathrm{g}$ ) Typical SEM ( $\mathrm{f}$ ) and TEM (g) images of monolayer $\mathrm{h}-\mathrm{BN}$ grown on $\mathrm{Cu}-\mathrm{Ni}$ alloy.
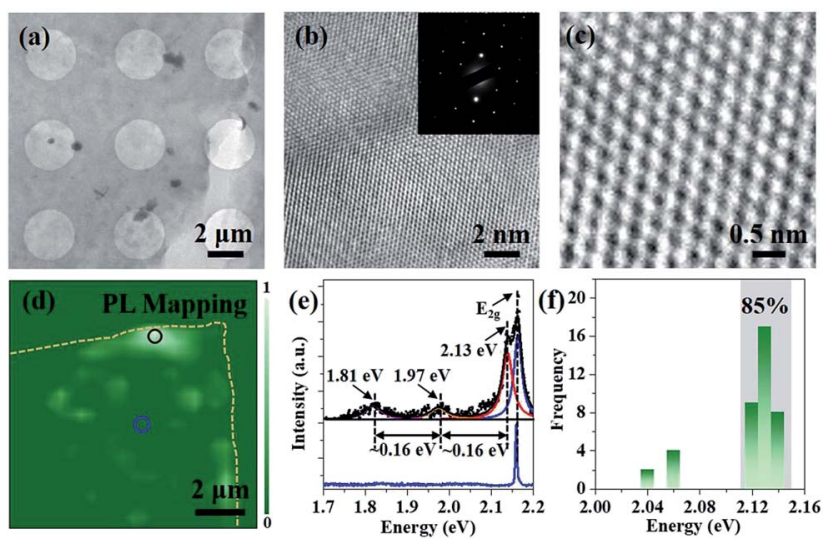

Fig. 4 (a) TEM image of $\mathrm{h}-\mathrm{BN}$ thin film suspended on Cu mesh grid. (b) High resolution TEM image of multilayer $h-B N$, the inset displays the SAED pattern. (c) Atomic resolution TEM image of multilayer $h-B N$. (d) Typical PL mapping of multilayer $\mathrm{h}-\mathrm{BN}$ on Si substrate. (e) Individual PL spectra extracted from the spots selected in d. (f) Histogram of h-BN zero phonon line (ZPL) energy generated from 40 emitters from $\mathrm{h}-\mathrm{BN}$ film. The shaded area highlights the range $E_{\text {emisson }}=(2.13 \pm 0.1) \mathrm{eV}$, which contains $85 \%$ of the emitters.

bulk h-BN. ${ }^{28}$ Moreover, as earlier theoretically and experimentally analysis, point defects would induce non-resonant absorption and emission within the band gap of materials. ${ }^{\mathbf{9} 29-31}$ Here, PL spectroscopy was used to investigate point defects distributions in h-BN film. ${ }^{\mathbf{9 , 3 0 , 3 1}}$ PL measurement was carried out at room temperature with a $532 \mathrm{~nm}$ sub-bandgap excitation laser. Fig. 4d illustrates the defect distributions in h-BN flake transferred on Si substrate. Optical emissions are accumulated on the edge of as-prepared h-BN multilayers, which may be introduced by the transfer and annealing processes. Typical PL spectra were extracted and fitted by Lorentzian fitting (Fig. 4e). Three peaks centred at $2.13 \mathrm{eV}, 1.97 \mathrm{eV}$ and $1.81 \mathrm{eV}$ were observed, which is consisted with zero phonon line (ZPL) and corresponding one- and two-optical-phonon sidebands (PSB), respectively. ${ }^{\mathbf{9}, 30,31}$ In addition, Fig. 4f displays a histogram of the emission energy values measured for 40 individual emitters. More than $85 \%$ of the emitters were measured to have a ZPL energy localized in a very narrow spectral window, $(2.13 \pm 0.1) \mathrm{eV}$. Such a small spectra variability of the ZPLs may be caused by strain and/or local lattice symmetry distortions. ${ }^{31}$ Moreover, it is observed that no optical emission was found on the large area of h-BN region, indicating the high quality of as-grown h-BN multilayers.

\section{Conclusion}

In this work, uniform and continuous multilayer h-BN has been obtained isothermally through the reaction between $\mathrm{Fe}_{2} \mathrm{~B}$ alloy and $\mathrm{N}_{2}$ via CVD method. The thickness could be mediated by controlling diffusion rate of nitrogen in the catalyst at different growth temperatures. Raman shift at $1366 \mathrm{~cm}^{-1}$ and sharp (0002) diffraction peak in the XRD pattern confirm the crystallinity of h-BN. TEM measurements revealed that the h-BN has highly orientated layered lattice structure. Comparing with 
monolayer h-BN growth on catalytic metal surfaces, this method shows its unique mechanism for achieving multilayer h-BN, which has potential on the synthesis of other layered 2D heterostructures. Therefore, the present achievement of highquality multilayer h-BN synthesis would give strong impact on ultrathin dielectric-, support-, or barrier-layer in particular for integrated electronics and photonics of 2D materials.

\section{Conflicts of interest}

There are no conflicts to declare.

\section{Acknowledgements}

We thank Xudong Hu for the Raman mapping of multilayer hBN. We also acknowledge Wei Liu and Ruxiang Shen for the XRD and XPS measurements. This work was financially supported by the National Key R\&D program (Grant No. 2017YFF0206106), the National Science Foundation of China (Grant No. 51772317), the Science and Technology Commission of Shanghai Municipality (Grant No. 16ZR1442700, 18511110700), Shanghai Rising-Star Program (A type) (Grant No. 18QA1404800), China Postdoctoral Science Foundation (Grant No. 2017M621563, 2018T110415).

\section{Notes and references}

1 K. S. Novoselov, Science, 2004, 306, 666-669.

2 K. F. Mak, C. Lee, J. Hone, J. Shan and T. F. Heinz, Phys. Rev. Lett., 2010, 105, 136805.

3 L. Li, F. Yang, G. J. Ye, Z. Zhang, Z. Zhu, W. Lou, X. Zhou, L. Li, K. Watanabe, T. Taniguchi, K. Chang, Y. Wang, X. H. Chen and Y. Zhang, Nat. Nanotechnol., 2016, 11, 593597.

4 K. Watanabe, T. Taniguchi and H. Kanda, Phys. Status Solidi, 2004, 201, 2561-2565.

5 A. K. Geim and I. V. Grigorieva, Nature, 2013, 499, 419-425. 6 L. Wang, I. Meric, P. Y. Huang, Q. Gao, Y. Gao, H. Tran, T. Taniguchi, K. Watanabe, L. M. Campos, D. A. Muller, J. Guo, P. Kim, J. Hone, K. L. Shepard and C. R. Dean, Science, 2013, 342, 614-617.

7 L. Britnell, R. V. Gorbachev, R. Jalil, B. D. Belle, F. Schedin, M. I. Katsnelson, L. Eaves, S. V. Morozov, A. S. Mayorov, N. M. R. Peres, A. H. Castro Neto, J. Leist, A. K. Geim, L. A. Ponomarenko and K. S. Novoselov, Nano Lett., 2012, 12, 1707-1710.

8 C. R. Dean, A. F. Young, I. Meric, C. Lee, L. Wang, S. Sorgenfrei, K. Watanabe, T. Taniguchi, P. Kim, K. L. Shepard and J. Hone, Nat. Nanotechnol., 2010, 5, 722726.

9 T. T. Tran, K. Bray, M. J. Ford, M. Toth and I. Aharonovich, Nat. Nanotechnol., 2016, 11, 37-41.

10 S. Kim, J. E. Fröch, J. Christian, M. Straw, J. Bishop, D. Totonjian, K. Watanabe, T. Taniguchi, M. Toth and I. Aharonovich, Nat. Commun., 2018, 9, 2623.

11 A. Sajid, J. R. Reimers and M. J. Ford, Phys. Rev. B, 2018, 97, 064101.
12 Y. Kubota, K. Watanabe, O. Tsuda and T. Taniguchi, Science, 2007, 317, 932-934.

13 S. Liu, R. He, Z. Ye, X. Du, J. Lin, H. Jiang, B. Liu and J. H. Edgar, Cryst. Growth Des., 2017, 17, 4932-4935.

14 Y. Shi, C. Hamsen, X. Jia, K. K. Kim, A. Reina, M. Hofmann, A. L. Hsu, K. Zhang, H. Li, Z.-Y. Juang, M. S. Dresselhaus, L.-J. Li and J. Kong, Nano Lett., 2010, 10, 4134-4139.

15 K. K. Kim, A. Hsu, X. Jia, S. M. Kim, Y. Shi, M. Hofmann, D. Nezich, J. F. Rodriguez-Nieva, M. Dresselhaus, T. Palacios and J. Kong, Nano Lett., 2012, 12, 161-166.

16 J. H. Park, J. C. Park, S. J. Yun, H. Kim, D. H. Luong, S. M. Kim, S. H. Choi, W. Yang, J. Kong, K. K. Kim and Y. H. Lee, ACS Nano, 2014, 8, 8520-8528.

17 S. Caneva, R. S. Weatherup, B. C. Bayer, B. Brennan, S. J. Spencer, K. Mingard, A. Cabrero-Vilatela, C. Baehtz, A. J. Pollard and S. Hofmann, Nano Lett., 2015, 15, 18671875.

18 S. M. Kim, A. Hsu, M. H. Park, S. H. Chae, S. J. Yun, J. S. Lee, D.-H. Cho, W. Fang, C. Lee, T. Palacios, M. Dresselhaus, K. K. Kim, Y. H. Lee and J. Kong, Nat. Commun., 2015, 6, 8662.

19 G. Lu, T. Wu, Q. Yuan, H. Wang, H. Wang, F. Ding, X. Xie and M. Jiang, Nat. Commun., 2015, 6, 6160.

20 A.-R. Jang, S. Hong, C. Hyun, S. I. Yoon, G. Kim, H. Y. Jeong, T. J. Shin, S. O. Park, K. Wong, S. K. Kwak, N. Park, K. Yu, E. Choi, A. Mishchenko, F. Withers, K. S. Novoselov, H. Lim and H. S. Shin, Nano Lett., 2016, 16, 3360-3366.

21 Y. Uchida, S. Nakandakari, K. Kawahara, S. Yamasaki, M. Mitsuhara and H. Ago, ACS Nano, 2018, 12, 6236-6244.

22 Q. Li, Q. Wu, J. Gao, T. Wei, J. Sun, H. Hong, Z. Dou, Z. Zhang, M. H. Rümmeli, P. Gao, J. Yan, J. Wang, J. Li, Y. Zhang and Z. Liu, Adv. Mater. Interfaces, 2018, 5, 1800662. 23 J. S. Lee, S. H. Choi, S. J. Yun, Y. I. Kim, S. Boandoh, J.-H. Park, B. G. Shin, H. Ko, S. H. Lee, Y.-M. Kim, Y. H. Lee, K. K. Kim and S. M. Kim, Science, 2018, 362, 817-821.

24 R. V. Gorbachev, I. Riaz, R. R. Nair, R. Jalil, L. Britnell, B. D. Belle, E. W. Hill, K. S. Novoselov, K. Watanabe, T. Taniguchi, A. K. Geim and P. Blake, Small, 2011, 7, 465468.

25 L. Schué, I. Stenger, F. Fossard, A. Loiseau and J. Barjon, $2 D$ Mater., 2016, 4, 015028.

26 Q. Cai, D. Scullion, A. Falin, K. Watanabe, T. Taniguchi, Y. Chen, E. J. G. Santos and L. H. Li, Nanoscale, 2017, 9, 3059-3067.

27 H. Tokoro, S. Fujii, T. Oku, T. Segi and S. Nasu, Mater. Trans., 2004, 45, 2941-2944.

28 Y. Kobayashi, T. Akasaka and T. Makimoto, J. Cryst. Growth, 2008, 310, 5048-5052.

29 K. Huang and A. Rhys, Proc. R. Soc. Lond. A Math. Phys. Sci., 1950, 204, 406-423.

30 N. R. Jungwirth and G. D. Fuchs, Phys. Rev. Lett., 2017, 119, 057401.

31 X. Li, G. D. Shepard, A. Cupo, N. Camporeale, K. Shayan, Y. Luo, V. Meunier and S. Strauf, ACS Nano, 2017, 11, 6652-6660. 